\title{
p53 mutation, but not in vitro predictor genes of therapeutic efficacy of cisplatin, is clinically relevant in comparing partial and complete responder cases of maxillary squamous cell carcinoma
}

\author{
ITSUHIRO KUDO ${ }^{1,2}$, MARIKO ESUMI $^{2}$, AKIHIRO KIDA $^{1}$ and MINORU IKEDA ${ }^{1}$ \\ Departments of ${ }^{1}$ Otorhinolaryngology-Head and Neck Surgery and ${ }^{2}$ Pathology, \\ Nihon University School of Medicine, 30-1 Ohyaguchikami-cho, Itabashi-ku, Tokyo 173-8610, Japan
}

Received March 9, 2010; Accepted April 27, 2010

DOI: $10.3892 /$ or_00000929

\begin{abstract}
To predict the efficacy of cisplatin and radiation therapy for maxillary squamous cell carcinoma, we examined the mRNA expression of 14 cisplatin-resistant genes and p53 mutation in specimens biopsied from patients prior to initiation of therapy. Five of 10 patients had mutations in the p53 gene, of whom four had residual tumors pathologically following chemoradiotherapy $(\mathrm{p}=0.0476)$. Of 14 genes examined, the mRNA expression of ATP7B was significantly lower in cases that were resistant to chemoradiotherapy. Six genes including multidrug resistance protein 1 (MDR-1), multidrug resistance associated protein 1 (MRP-1), $\mathrm{Cu}^{++}$transporting, $\beta$ polypeptide (ATP7B), xeroderma pigmentosum, complementation group A (XPA), excision repair cross-complementing rodent repair deficiency, complementation group 1 (ERCC-1) and B-cell CLL/lymphoma 2 (BCL2) were down-regulated in cases of recurrent cancers. These results show that the evaluation of p53 mutation provides the most useful predictor of therapeutic effects. In responder cases, the drug-resistant genes that were determined in cell lines by culture do not necessarily translate into clinical relevance.
\end{abstract}

\section{Introduction}

Cisplatin is currently one of the most widely used anticancer drugs in the world. Its action is initiated by migration of cisdiamine-dichloroplatinum into the nucleus of cancer cells and binding to the nitrogen at position 7 of guanine, resulting in cell cycle arrest and apoptosis (1). Platinum-based cancer chemotherapy is currently one of the main anticancer drugs for the treatment of maxillary squamous cell carcinoma. A

Correspondence to: Dr Mariko Esumi, Department of Pathology, Nihon University School of Medicine, 30-1, Ohyaguchikami-cho, Itabashi-ku, Tokyo 173-8610, Japan

E-mail:mesumi@med.nihon-u.ac.jp

Key words: p53 mutation, maxillary squamous cell carcinoma, cisplatin, drug-resistance combined therapy of platinum drugs with radiotherapy and surgery has been shown to improve the therapeutic results. However, the occurrence of therapy-resistant tumors is a major problem. Even after resective surgery, the tumors often recurred. Thus, there is a range of therapeutic responses and varying degree of resistance in maxillary squamous cell carcinoma. It is important for the development of new and improved therapies to clarify the mechanisms of drugresistance of cancers.

Recently, the pathway from platinum-DNA adducts to ultimate apoptosis has been gradually elucidated by using cisplatin-resistant cell lines (1-7). The involvement of processes such as efflux of anticancer drugs, detoxification of anticancer drugs, DNA repair and inhibition of apoptosis has been considered as mechanisms contributing to the resistance of cisplatin in cancer (Fig. 1). With regard to cisplatin efflux, the expression of ATPase, $\mathrm{Cu}^{++}$transporting, $\mathrm{B}$ polypeptide (ATP7B) may be a contributing factor $(1,7)$. The conjugate of cisplatin with intracellular glutathione, catalyzed by the enzyme glutathione S-transferase $\pi$ (GST $\pi$ ), is readily exported from cells by export pumps such as multidrug resistance associated protein 1 (MRP1) and multidrug resistance associated protein 2 (MRP2) (1). Decreases in pyruvate kinase and muscle $2(P K-M 2)$, and increases in activating transcription factor 4 (ATF4) were found to correlate with cisplatin resistance $(3,5)$. In addition, up-regulation of DNA repair genes such as excision repair cross-complementing rodent repair deficiency, complementation group 1 (ERCC1) and xeroderma pigmentosum, complementation group A (XPA) may also contribute to the development of cisplatin-resistance $(4,8)$. Inhibition of apoptosis, which is caused by upregulation of B-cell CLL/lymphoma 2 (BCL2) (6) or downregulation of apoptotic peptidase activating factor 1 (APAF1) (2), BCL2-associated X protein (BAX) (9) and Fas ligand (FASL) (10), is also associated with cisplatin resistance. These findings were observed in vitro using cisplatinresistant cell lines. It is crucial to explore whether similar mechanisms of tumor resistance to cisplatin are applicable in vivo in carcinoma tissues, but thus far, few studies have been performed (9-11). In this study, we examined the mRNA expression of 14 candidate genes and the prevalence of $p 53$ mutation in patients with maxillary squamous cell carcinoma, and analyzed the data with reference to therapeutic results. 


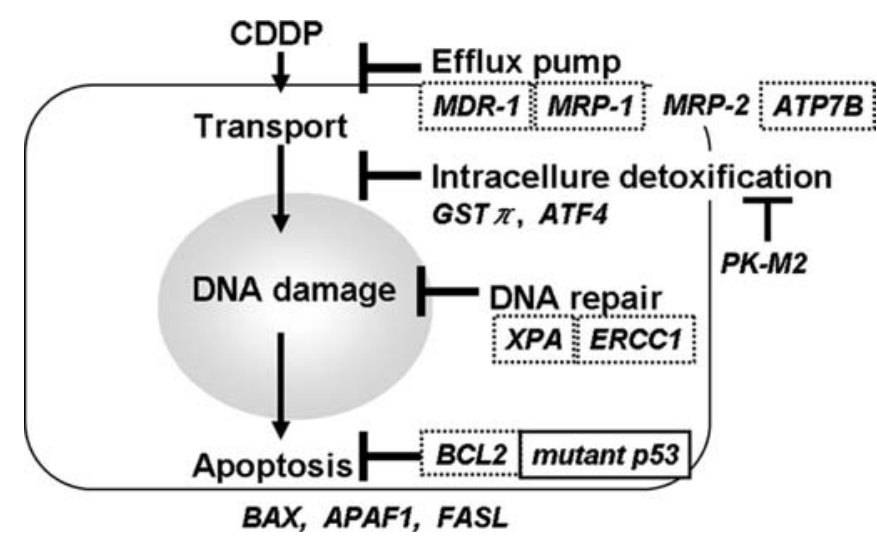

Figure 1. Candidate genes relevant to tumor resistance to cisplatin. Four major steps that are considered mechanisms in the resistance to anticancer drugs and the candidate genes that may be relevant to resistance to cisplatin are illustrated. Italicized gene symbols relevant to each step are explained in the text. The gene symbols with full-line box, p53 gene mutations that are correlated with resistance to chemoradiotherapy. The gene symbols with dotted-line boxes, drug-resistant genes whose mRNA expressions were negatively correlated with resistance to chemoradiotherapy.

\section{Materials and methods}

Patients. Tissue samples were obtained from 10 patients with maxillary squamous cell carcinoma (Table I). Maxillary tissues were biopsied upon the initial presentation, and they were fixed with formalin for histopathological studies and cryopreserved at $-80^{\circ} \mathrm{C}$. For treatment of maxillary cancer, all patients underwent concurrent chemotherapy and radiotherapy. One course of chemotherapy included cisplatin administration on day 1 at a dose of $100 \mathrm{mg} / \mathrm{kg}$ and $5-\mathrm{FU}$ administration from day 2 to 6 at a dose of $1000 \mathrm{mg} / \mathrm{kg} / \mathrm{day}$. All patients received a total of 5 chemotherapy courses with intervals of 2 to 4 weeks. Radiation therapy was initiated with the third course of chemotherapy. All patients underwent biopsies for the evaluation of therapeutic effectiveness after the treatment with a total dose of 40 Gy of radiation. Cases with biopsy results negative for cancer cells received a subsequent radiotherapy course with total doses up to $60 \mathrm{~Gy}$, and cases with positivity for cancer cells underwent surgical removal of residual tumors. At the completion of chemoradiotherapy, all patients showed clinical regression of tumors and were followed-up to check for recurrence. The TNM staging system of the International Union Against Cancer was used for the classification of stage and grade of malignant tumors (12). Informed consent was obtained from all study subjects.

Quantification of $m R N A$. Total RNA was extracted using TRIzol (Invitrogen, Carlsbad, CA, USA) according to the instruction of the manufacturer. The quality of RNA was evaluated by Agilent RNA 6000 Nano Assay Reagent Kit (Agilent Bioanalyzer, Agilent Technologies, Palo Alto, CA, USA), and specimens with two distinct bands (28s and 18s rRNA) were selected for analysis. Total RNA was treated with DNase I (Takara Shuzo, Kyoto, Japan) at $37^{\circ} \mathrm{C}$ for $20 \mathrm{~min}$ and purified with the TRIzol reagent. DNase-treated RNA $(10 \mu \mathrm{g})$ was used to synthesize cDNA with random primers and AMV reverse transcriptase XL (Life Sciences, Gaithersburg, MD, USA) in a total volume of $100 \mu 1$.

Real-time RT-PCR for quantification of gene expression was performed using the Rotor-Gene 3000 (Corbett Research, Mortlake, Australia) and ABI Prism 7000 Sequence Detection

Table I. Clinicopathological features of patients with maxillary squamous cell carcinoma.

\begin{tabular}{|c|c|c|c|c|c|c|c|c|}
\hline \multirow[b]{2}{*}{ Case } & \multirow[b]{2}{*}{ Age } & \multirow[b]{2}{*}{ Stage } & \multirow[b]{2}{*}{ Grade } & \multirow[b]{2}{*}{ Histology ${ }^{a}$} & \multicolumn{2}{|c|}{ Recurrence $^{b}$} & \multicolumn{2}{|c|}{ p53 } \\
\hline & & & & & - & + & Mutation $^{c}$ & $\mathrm{mRNA}^{\mathrm{d}}$ \\
\hline M11 & 58 & III & 2 & $\mathrm{~N}$ & $84 \mathrm{M}$ & & & 1.48 \\
\hline M5 & 64 & III & 3 & $\mathrm{~N}$ & $74 \mathrm{M}$ & & & 15.25 \\
\hline $7 \mathrm{~T}$ & 60 & III & 2 & $\mathrm{~N}$ & $60 \mathrm{M}$ & & & 8.49 \\
\hline M2 & 55 & III & 2 & $\mathrm{~N}$ & $55 \mathrm{M}$ & & & 7.84 \\
\hline M6 & 55 & III & 1 & $\mathrm{~N}$ & & $9 \mathrm{M}$ & & 3.87 \\
\hline M12 & 80 & IV A & 2 & $\mathrm{~N}$ & $(1 \mathrm{M})$ & & c. $782+1 \mathrm{G} \rightarrow \mathrm{A}$ & 2.81 \\
\hline M9 & 73 & III & 1 & $\mathrm{~T}$ & $46 \mathrm{M}$ & & P190T & 22.56 \\
\hline M13 & 51 & III & 3 & $\mathrm{~T}$ & & $6 \mathrm{M}$ & 156delFS & 2.46 \\
\hline M1 & 63 & III & 2 & $\mathrm{~T}$ & & $4 \mathrm{M}$ & R280S & 3.00 \\
\hline M10 & 64 & III & 2 & $\mathrm{~T}$ & & $2 \mathrm{M}$ & c. $782+1 \mathrm{G} \rightarrow \mathrm{A}$ & 4.74 \\
\hline
\end{tabular}

${ }^{a}$ Histopathological examination results on biopsied specimens for the evaluation of therapeutic effectiveness at the termination of radiation therapy with a total dose of $40 \mathrm{~Gy}$. N, negative for tumor; T, positive for tumor. ${ }^{b}$ The symbol minus (-) illustrates no recurrence of tumor for follow-up months after completion of chemoradiotherapy. The symbol positive (+) indicates recurrence. The case M12 should be excluded for evaluation of recurrence because the follow-up was ceased one month after completion of chemoradiotherapy. c.782+1G $\rightarrow \mathrm{A}, \mathrm{splicing}$ defect due to point mutation of $\mathrm{G} \rightarrow \mathrm{A}$ at the first base of intron 7; P190T, missense mutation of proline to threonine at codon 190 due to point mutation; R280S, missense mutation of arginine to serine at codon 280; 156delFS, frameshift at codon 156 due to one base deletion. ${ }^{\mathrm{d}} \mathrm{mRNA}$ quantity expressed by normalization with that of an internal control gene, GAPDH. 
Table II. Primer sequences used in this study.

\begin{tabular}{|c|c|c|c|}
\hline \multirow[b]{2}{*}{ Function } & \multirow[b]{2}{*}{ Gene } & \multicolumn{2}{|c|}{ Primer sequence } \\
\hline & & Forward (5'-3') & Reverse $\left(5^{\prime}-3^{\prime}\right)$ \\
\hline \multirow[t]{4}{*}{ Efflux pump } & MDR-1 & GCTCAGACAGGATGTGAGTT & TTACAGCAAGCCTGGAACCT \\
\hline & MRP-1 & TACCTCCTGTGGCTGAATCT & GGGCCACCTGATACGTCTTG \\
\hline & MRP-2 & TTCAGCGAGACCGTATCAGG & ACCTGTTGGAGGTGATCCAG \\
\hline & ATP7B & ACTGGTGGAAGAGGCTCAGA & GTGCTTGTTGGGGTTAGGAA \\
\hline \multirow{3}{*}{$\begin{array}{l}\text { Intracellular } \\
\text { detoxication }\end{array}$} & $\mathrm{GST} \pi$ & GGCAACTGAAGCCTTTTGAG & TCATGGATCAGCAGCAAGTC \\
\hline & ATF4 & TGACCACGTTGGATGACACT & CCTGGTCGGGTTTTGTTAAA \\
\hline & PK-M2 & GAGTACCATGCGGAGACCAT & GCGTTATCCAGCGTGATTTT \\
\hline \multirow[t]{2}{*}{ DNA repair } & XPA & GCAGCCCCAAAGATAATTGA & TGGCAAATCAAAGTGGTTCA \\
\hline & ERCC1 & ATGTGAAAGATCCCCAGCAG & GCTGGTTTCTGCTCATAGGC \\
\hline \multirow[t]{6}{*}{ Apoptosis } & p53 (1) & AACAACACCAGCTCСТСТCC & CTCTCGGAACATCTCGAAGC \\
\hline & p53 (2) & CATTCTGGGACAGCCAAGTC & CTCTCGGAACATCTCGAAGC \\
\hline & BAX & ATGGAGCTGCAGAGGATGAT & AACAACACCAGCTCCTCTCC \\
\hline & APAF1 & GGGACTCTTGGGTGTTGAAA & TTCTGGCAAATCTGCCTTCT \\
\hline & FASL & GCACTTTGGGATTCTTTCCA & CCTCCATTTGTCTGGCTCAT \\
\hline & BCL2 & TTGTTCAAACGGGATTCACA & GGCTGGGCACATTTACTGTT \\
\hline Control & GAPDH & GGTCGGAGTCAACGGATTTG & GGATCTCGCTCCTGGAAGAT \\
\hline
\end{tabular}

System (Applied Biosystems, Foster, CA, USA). The realtime PCR assay was carried out using SYBR-Green Realtime PCR Master Mix (Applied Biosystems) with 10 ng cDNA and $0.5 \mu \mathrm{M}$ of each primer in $25 \mu 1$ volume of solution. Thermal cycling conditions were as follows: the reaction mixture was preheated at $95^{\circ} \mathrm{C}$ for $10 \mathrm{~min}, 45$ cycles of $95^{\circ} \mathrm{C}$ for $15 \mathrm{sec}$, and $60^{\circ} \mathrm{C}$ for $60 \mathrm{sec}$. The primers used are shown in Table II. Standard samples were prepared by 5-fold serial dilution of cDNA obtained from a maxillary squamous cell carcinoma to generate standard curves. Gene expression was determined by the relevant value normalized by the internal control gene, glyceraldehyde-3-phosphate dehydrogenase (GAPDH).

p53 mutation analysis. PCR amplification of the hotspot mutation region (aa115-aa342) of the $p 53$ gene was performed using the cDNA described above. The primers used are shown in Table II. When two PCR product bands (687 and 1029 bp) were detected following electrophoresis, each was extracted from the agarose gels and purified separately using QIAquick Gel Extraction Kit (Qiagen, Hilden, Germany). Otherwise, PCR products were purified using SigmaSpin (Sigma-Aldrich, St. Louis, MO). DNA sequencing was performed with the Big Dyeterminator kit (Applied Biosystems) and an automated sequence system ABI PRISM 310 Genetic Analyzer (Applied Biosystems).

Statistical analysis. Therapeutic results and recurrence rate were statistically evaluated with reference to the patients' age using Student's t-test, and the background factors including clinical stage and histopathological malignancy grade of maxillary squamous cell carcinoma were analyzed using Mann-Whitney U test. P-values $<0.05$ were considered statistically significant. Likewise, correlation of gene expression levels with therapeutic effectiveness and recurrence rate was determined using Mann-Whitney U test. Furthermore, Fischer's exact test for $2 \times 2$ contingency tables was used to test association of p53 mutations with therapeutic effectiveness and recurrence rate.

\section{Results}

Therapeutic effectiveness. Table I summarizes the chemoradiotherapy results in 10 patients with maxillary squamous cell carcinoma. Tissue biopsies at the termination of radiation therapy with a total dose of 40 Gy revealed regression of tumors in six cases and residual tumors in four cases. The four cases with residual tumors underwent surgical excision, but one of the six negative cases and three with residual tumors had recurrent disease within one year after therapy. Correlation analysis between resistance to the chemoradiologic therapy and the patients' age, clinical stage, or differentiation grade of tumors did not reveal any statistical significance.

p53 mutation. We determined the nucleotide sequence of the mutational hotspot region (exon 4 to exon 10) of the p53 gene, as shown in Fig. 2 and Table II. Five of the 10 cases of maxillary squamous cell carcinoma showed mutations 


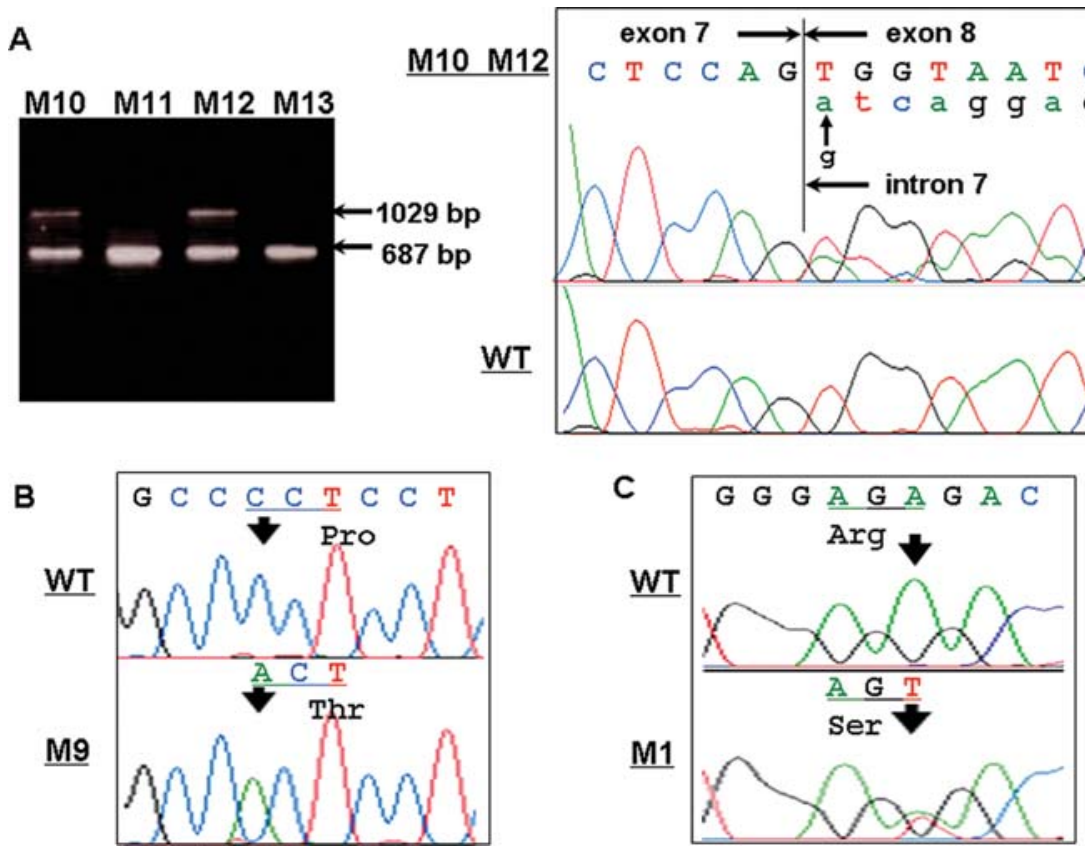

Figure 2. p53 mutations of cases M1, M9, M10 and M12. (A) Agarose gel electrophoreses of p53 RT-PCR products show an abnormal 1029 bp fragment in M10 and M12 (left). Sequencing of PCR products from M10 and M12 shows a G $\rightarrow$ A point mutation in the first base of intron 7 of $p 53$ (right). WT, wild-type. (B) $p 53$ sequence of $\mathrm{M} 9$ illustrates $\mathrm{C} \rightarrow \mathrm{A}$ transversion that resulted in missense mutation from proline (Pro) to threonine (Thr). (C) $p 53$ sequence of M1 illustrates $\mathrm{A} \rightarrow \mathrm{T}$ transversion that resulted in missense mutation from arginine (Arg) to serine (Ser).

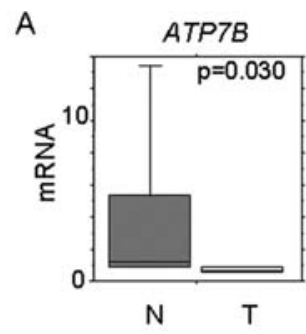

B
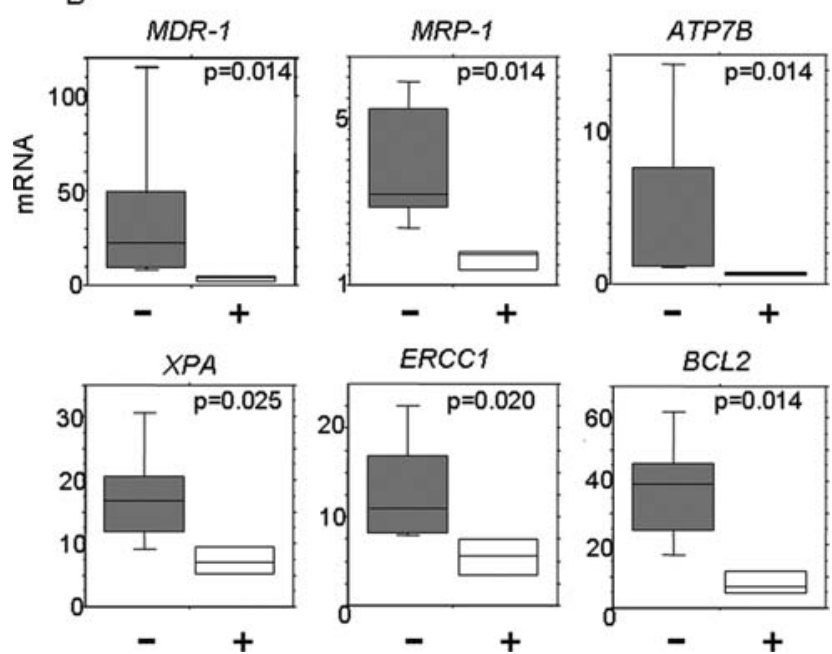

Figure 3. Comparison of mRNA levels of drug-resistant genes between the chemoradiotherapy-sensitive and -resistant groups with maxillary squamous cell carcinoma. The mRNA expression shows quantitative values normalized by the expression of an internal control gene, GAPDH. The difference between the 2 groups is statistically significant in the expression of these genes. (A) mRNA level of ATP7B was compared between six tumor-free (N) and four tumor-positive (T) cases during the course of chemoradiotherapy. $\mathrm{p}=0.030$. (B) mRNA levels of six genes were compared between five successful chemoradiotherapy (-) and four recurrent cases (+) within six months after treatment. in this gene region. The cases M10 and M12 had a common splicing defect, which resulted in a 1029 bp fragment in addition to the wild-type $687 \mathrm{bp}$ fragment, and sequencing revealed transcription of 342 bp from intron 7 in these 2 cases (Fig. 2A). Other mutations included frameshift (M13) and point mutation causing a single amino acid alteration (M1, M9) (Fig. 2B and C). Noticeably, in the case M9 only the mutant-type gene was identified, but not the wild-type gene (Fig. 2C). In contrast, the 5 cases with wild-type $p 53$ had no abnormality in the quantity as well as the quality of $p 53$ gene expression (Table I). With regard to the correlation between the p53 mutations and chemoradiotherapy resistance, there was a significant correlation between the $p 53$ gene mutations with therapeutic effectiveness $(\mathrm{p}=0.0476)$, but not with the recurrence of malignant lesions after treatment $(p=0.2603)$.

mRNA expression of drug-resistant genes. We measured the mRNA levels of representative genes that may be involved in the four mechanisms of tumor resistance to anticancer drugs, as shown in Fig. 1 and Table II. Only the ATP7B gene among the 14 genes examined was significantly down-regulated in the chemoradiotherapy-resistant group (Fig. 3A). Six genes were significantly down-regulated in the group with recurrent maxillary squamous cell carcinoma (Fig. 3B). Genes with altered mRNA expression are marked in Fig. 1.

\section{Discussion}

To clarify the epigenetic difference between patients with partial and complete responses to chemoradiotherapy, in this study, we focused on the gene expression of potential therapy-resistant genes and the mutational status of p53 in responder subjects with maxillary squamous cell carcinoma. 
In recent years, studies on the mechanisms of carcinoma cell resistance to cisplatin have focused on the mutation status of the $p 53$ gene. The $p 53$ protein has a nuclear localization signal (NLS) in amino acid residues 305 to 322 , which is necessary for nuclear translocalization and activation of p53 function. p53 functions as a tumor suppressor upon damage to DNA by transciptionally inducing cyclin inhibitors, repair enzymes and/or proapoptotic genes, resulting in cell cycle arrest, repair of DNA damage and/or apoptosis, respectively. Alterations to the NLS might cause difficulty in the nuclear translocalization of $\mathrm{p} 53$, resulting in a decrease or inhibition of these functions (13). Mandic et al showed that loss of p53 NLS correlates with resistance to cisplatin in experiments using cell lines of head and neck squamous cell carcinoma in culture (14). In the present study, three cases (M10, M12, M13) were confirmed to have abnormal NLS, and these three cases had poor prognosis probably because of reduced apoptosis of the carcinoma cells due to p53 mutations. It is remarkable that one of the cases (M12) had a p53 mutation and yet it was tumor-free according to the biopsies; a possible explanation is failure of detection of residual tumors because of sampling error. In any event, it is plausible that cisplatin induces apoptosis of carcinoma cells by direct interaction with tumor cell DNA and that p53 mediates cisplatin in the suppression of cancer progression. Further studies using integral analyses of various relevant gene expression in maxillary squamous cell carcinoma are required to elucidate the genes that are involved in the mechanisms of tumor resistance to chemoradiotherapy.

This study shows mutations in the $p 53$ gene in $50 \%$ of patients with maxillary squamous cell carcinoma. Even in the cases (M1 and M9) with a single amino acid alteration, the mutations were present in the DNA binding domain that renders it non-functional (IARC TP53 DATABASE). Thus, all mutations of the $p 53$ gene examined in this study cause loss-of-function. A number of previous studies on the prevalence of $p 53$ alterations in patients with head and neck squamous cell carcinoma described a range of $44-68 \%$ of cases with the disease (15-18). Bosch et al showed that the prevalence of $p 53$ alterations varies in different sites of head and neck squamous cell carcinoma, where it is significantly higher in hypopharyngeal tumors $(66 \%)$, than in the other sites $(38 \%)$ (19). However, few reports have addressed the prevalence of $p 53$ mutations in maxillary squamous cell carcinoma. Our observations suggest that the prevalence of $p 53$ mutations in maxillary squamous cell carcinoma ranks the highest among other head and neck squamous cell carcinomas, excepting hypopharynx tumors. It has widely been recognized that the sites of $p 53$ gene mutations are predominant in exons 4-9 in a diverse category of carcinomas (16). The current study is also in agreement with the general view that the majority of $p 53$ mutations occur in exons 5,6 , and 8 , and intron 7 , as described previously. It is noticeable that the relevant p53 mutations are of the loss-of-function type that causes inactivation of the $\mathrm{p} 53$ protein. Furthermore, our patients with mutant $p 53$ had a poor response to concurrent cisplatin and radiotherapy with a significantly higher frequency of residual tumor occurrence at the time of evaluation of chemoradiotherapeutic effectiveness $(\mathrm{p}=0.0476)$, the $p 53$ mutation rate was $100 \%(4 / 4)$ in cases with residual tumors and only $17 \%(1 / 6)$ in cases of successful chemoradiotherapy. Cabelguenne et al studied p53 alterations in head and neck squamous cell carcinoma that were divided into 2 groups, responders and non-responders to neoadjuvant chemotherapy. They found that the prevalence of $p 53$-mutated tumors was higher in the group of non-responders than responders (81 versus $50 \%$, respectively) (16). In our study, we only examined the responders to neoadjuvant chemotherapy, and divided them into partial and complete responders. We found that p53 mutation prevalence was still much higher in the group of partial responders than in complete responders.

In the current study, we could not identify any chemotherapy-resistant genes that were highly expressed in cases of residual tumors following chemoradiotherapy. Further studies that directly examine the mRNA expression profile in the residual tumor tissues resistant to the therapy will enable us to elucidate the relevant genes involved. In contrast, we found that the mRNA expression of a certain group of genes was instead reduced and shown to correlate with resistance to chemoradiotherapy (Fig. 3B). It is yet unknown why the genes were significantly down-regulated in the cases of tumor resistance. Miyazaki et al reported that neither BCL2 nor BAX mRNA expression in tissue samples of esophageal squamous cell carcinoma was related to the efficacy of cisplatin therapy (11). Their findings suggest that there is a difference between studies using cell lines (6) and human tissues. Further studies are necessary to facilitate understanding of tumor resistance to chemotherapy not only in cell lines, but also in clinical tissue samples.

\section{References}

1. Kelland L: The resurgence of platinum-based cancer chemotherapy. Nat Rev Cancer 7: 573-584, 2007.

2. Kamarajan P, Sun NK, Sun CL and Chao CC: Apaf-1 overexpression partially overcomes apoptotic resistance in a cisplatinselected HeLa cell line. FEBS Lett 505: 206-212, 2001.

3. Tanabe M, Izumi H, Ise $\mathrm{T}$, et al: Activating transcription factor 4 increases the cisplatin resistance of human cancer cell lines. Cancer Res 63: 8592-8595, 2003.

4. Wu X, Fan W, Xu S and Zhou Y: Sensitization to the cytotoxicity of cisplatin by transfection with nucleotide excision repair gene xeroderma pigmentosun group A antisense RNA in human lung adenocarcinoma cells. Clin Cancer Res 9: 5874-5879, 2003.

5. Yoo BC, Ku JL, Hong SH, et al: Decreased pyruvate kinase M2 activity linked to cisplatin resistance in human gastric carcinoma cell lines. Int J Cancer 108: 532-539, 2004.

6. Cho HJ, Kim JK, Kim KD, et al: Upregulation of Bcl-2 is associated with cisplatin-resistance via inhibition of Bax translocation in human bladder cancer cells. Cancer Lett 237: 56-66, 2006.

7. Mangala LS, Zuzel V, Schmandt R, et al: Therapeutic targeting of ATP7B in ovarian carcinoma. Clin Cancer Res 15: 3770-3780, 2009.

8. Olaussen KA, Mountzios G and Soria JC: ERCC1 as a risk stratifier in platinum-based chemotherapy for non-small-cell lung cancer. Curr Opin Pulm Med 13: 284-289, 2007.

9. Sugimoto C, Fujieda S, Seki M, et al: Apoptosis-promoting gene (bax) transfer potentiates sensitivity of squamous cell carcinoma to cisplatin in vitro and in vivo. Int J Cancer 82: 860-867, 1999.

10. Fujieda S, Sunaga H, Tsuzuki H, et al: Expression of fas (CD95) ligand is correlated with IL-10 and granulocyte colony-stimulating factor expression in oral and oropharyngeal squamous cell carcinoma. Cancer Lett 161: 73-81, 2000.

11. Miyazaki T, Kato H, Faried A, et al: Predictors of response to chemo-radiotherapy and radiotherapy for esophageal squamous cell carcinoma. Anticancer Res 25: 2749-2755, 2005.

12. Sobin L and Wittekind C: TNM Classification of Malignant Tumours. 6th edition. Wiley-Liss, New York, 2002. 
13. Shaulsky G, Goldfinger N, Tosky MS, Levine AJ and Rotter V: Nuclear localization is essential for the activity of p53 protein. Oncogene 6: 2055-2065, 1991.

14. Mandic R, Schamberger CJ, Muller JF, et al: Reduced cisplatin sensitivity of head and neck squamous cell carcinoma cell lines correlates with mutations affecting the $\mathrm{COOH}$-terminal nuclear localization signal of p53. Clin Cancer Res 11: 6845-6852, 2005

15. Sakai E, Rikimaru K, Ueda M, et al: The p53 tumor-suppressor gene and ras oncogene mutations in oral squamous-cell carcinoma. Int J Cancer 52: 867-872, 1992.

16. Cabelguenne A, Blons H, de Waziers I, et al: p53 alterations predict tumor response to neoadjuvant chemotherapy in head and neck squamous cell carcinoma: a prospective series. J Clin Oncol 18: 1465-1473, 2000.
17. Weber A, Bellmann U, Bootz F, Wittekind C and Tannapfel A: INK4a-ARF alterations and p53 mutations in primary and consecutive squamous cell carcinoma of the head and neck. Virchows Arch 441: 133-142, 2002.

18. Eriksen JG, Alsner J, Steiniche T and Overgaard J: The possible role of TP53 mutation status in the treatment of squamous cell carcinomas of the head and neck (HNSCC) with radiotherapy with different overall treatment times. Radiother Oncol 76: 135-142, 2005.

19. Bosch FX, Ritter D, Enders C, et al: Head and neck tumor sites differ in prevalence and spectrum of p53 alterations but these have limited prognostic value. Int J Cancer 111: 530-538, 2004. 